\title{
Intent Classification using BERT for Chatbot application pertaining to Customer Oriented Services
}

\author{
Dr Geetha . N, Mr Vivek G, Vinetia T A \\ \{*sng.mca@psgtech.ac.in, vivek007psg@gmail.com, venetiaadbe@gmail.com\}
}

Assistant Professor (Selection Grade), Department of Computer Applications, PSG College of Technology, Tamilnadu, Coimbatore-641004, India ${ }^{1}$,PG Student, Department of Computer Applications, PSG College of Technology, Tamilnadu, Coimbatore-641004, India ${ }^{2,3}$

\begin{abstract}
In current scenario, customer interactions with live chat interfaces are very popular and real time customer service are offered in a better way. Traditional human chat services are replaced by conversational agents. The conversational agents or chat bots had become an integral part of business for customer services. Chat bots are the software designed to perform textual or audio conversations with the customers/users. Chat bots provide services with less time but at times fail to meet the customer expectations. Initial days, chat bots are designed by using simple pattern matching and string processing methods using rule-based models. Recently AI based chat bots using machine learning algorithms are developed to handle conversation context. To be more interactive like humans, Conversational AI chat bots are developed with Natural Language Processing (NLP) and Natural Language Understanding (NLU). BERT Model and go Bot are used to identify intent by chat bot and tested using dataset pertaining to networking domain. The results show that the accuracy of conversation is better and can be improved for large dataset.
\end{abstract}

Keywords: BERT, intent, chat bot, neural network, contextual, go Bot

\section{$1 \quad$ Introduction}

Intent Classification is an important task in Natural Language Understanding(NLU). In recent days, chatbots are placing its footprints in business world. Chatbot is one of the services which serves customer 24 hours a day, 7days a week and 365 days a year. Simple Chatbot will answer only the particular questions and not according to the user's need. They are developed only on particular goal and it cannot answer all the queries the customer need and they are key based. To overcome this entire problem, Conversational Artificial Intelligence (AI) comes to aid. The Conversational AI Chatbot is developed to make life easier for the enterprises and customer. Conversational AI Chatbot are developed using NLP(Natural Language Processing), NLU(Natural Language Understanding) and other components which makes the Chatbot more interactive and behave like a human.

Deep Learning is used to create Chatbots in different domains and Chatbot will help the user with correct solution for the problem mentioned by the user in an efficient manner.The objective of this paper is to develop a Contextual Chatbot which uses Machine Learning and Artificial Intelligence to remember conversations with specific users to learn and grow over time and satisfy their needs. The challenging part of the Chatbot is to find the key state of the conversation. Key State is found for the bot to remember the previous conversation and reply accordingly. To overcome this challenge Natural Language Processing, Natural Language Understanding, Natural Language Generation Techniques are used. 


\section{Related Work}

Many researchers contributed in the field of chatbot development. Chatbot applications are classified as commercial, entertainment, service and advisory[1]. In otherway, chatbot applications can be task oriented and non task oriented[2]. Different algorithms and techniques are involved in developing chatbot. The idea of using DNN to focus on various parts of input was applied in neural translation[3]. Sutskever et al [4] used multilayered Long Short-Term(LSTM) approach for translating long sentences. Single LSTM is used to map a vector of fixed dimension and added deep LSTM to decrypt target sequences from the given vector. AI based functional capabilities[5][6] for natural language processing and understanding as dialogue statement was offered by IBM Watson assistant cloud services. Also AI based chatbot[7] is developed for customer self-service context to fulfil then customer needs.

\section{Proposed Work}

This project is mainly focused in building contextual goal oriented chatbot. Contextual Chatbotcan understand the meaning and respond to the user accordingly and can differentiate the meaning of each utterance given by the user.The two main components while building a contextual chatbot are NLU (Natural Language Understanding) and NLG (Natural Language Generation)

NLU teaches machines how to interpret and understand language which is given by the user. The aim is to make computers identify what a body of text or spoken speech means. NLU uses AI algorithms to recognize the attribute of language which is sentiment, semantics, context, intent and entities. The different task done by NLU is Relational Extraction, Semantic Parsing, Dialogue Agents, Question \& Answering and Summarization This system will differentiate the intent and understand the meaning of the sentence and answer accurately so this make customer to think that they are having conversation with human. In this work, two models like BERT model and GoBoT model are adopted to find the intent.

Architectural Model

The first model is Intent classification which classifies the intent based on query asked by the customer. For intent classification, the BERT model is used. For a chatbot with NLU, NLG DM, DS the architecture is shown in Fig 1.

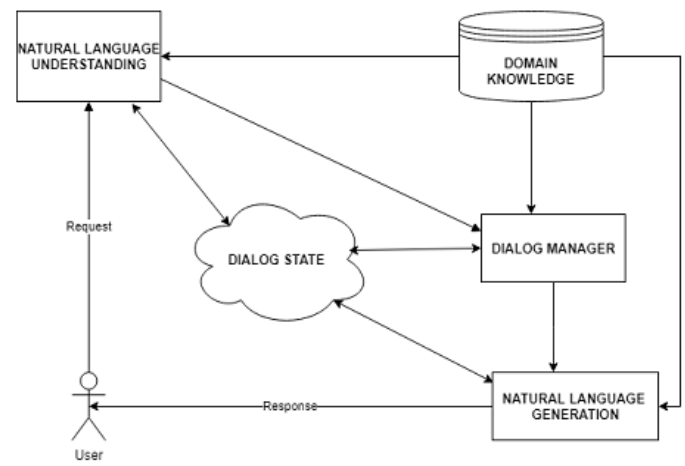

Fig. 1. Chatbot Architecture

The NLU is responsible for transforming the user utterances to a predefined semantic frame that the system can understand. The main task of NLU is to classify the intent and slot filling from the user's conversation. Intent classification is one of the important tasks in any chatbot this makes the life easier and answer accurately to the queries asked by the user.

Dialog state must store the current intent topic (Dialog Act) of each utterance. It must also remember the previous intent. In order to implement this different data structure is used like hashing, dictionary etc. Dialog state must keep track of information relevant to the dialog and the dialog state 
must be updated throughout the conversation. In fig 1 the Dialog state is typically stored in some frame data structure like hash table, dictionary etc., and is designed to be continuously updated throughout the duration of the conversation.

Dialog manager will help to find the dialog foci which mean the solution to the problem. NLG must respond to the user according to the dialog state and dialog manager. Dialog manager will structure the response according to the current user input and Dialog State. The task of Dialog Manager is to initialize the problem identification, grounding i.e. process of finding the solution to the given request.

NLG is a process where structured data is transformed into Natural Conversational Language for output to the user. With the help of NLG, data from state machine are taken and then turned into unstructured data. The output will be in the form of Human Language.

The second model is the GoBot model from Deeppavlov which include Intent classification, Slotfill and embedders shown in fig 2. There are different framework which provides these facilities in python. One of the frameworks is Deeppavlov. Deeppavlov provides pre-trained NLP models and predefined Dialog system which is very important in building contextual chatbot. Deeppavlov also provide a framework for implementing and testing own dialog system. Building a contextual chatbot is complex. It needs framework to support the working of a chatbot.

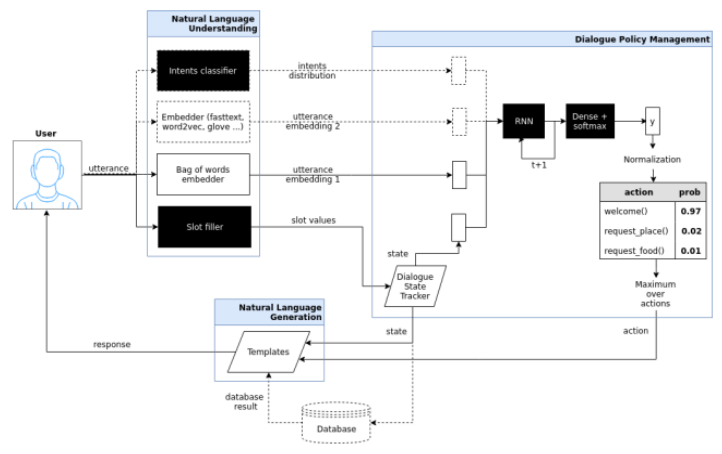

Fig. 2 GoBot Model

In fig 2 the dialog policy management is the main component which holds the chatbot model firmly. In this model Recurrent Neural Network is implemented.The flow of the chatbot is shown in fig 3. 


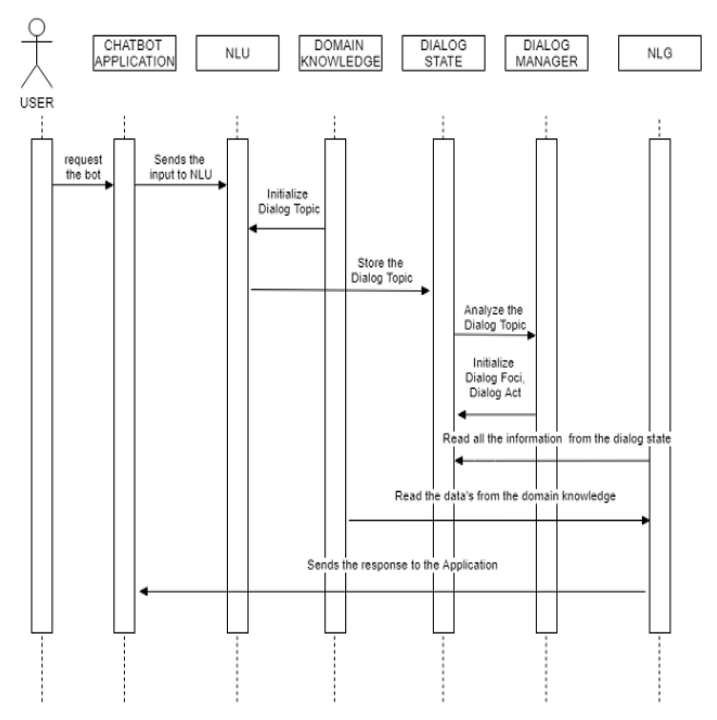

Fig 3 System flow of chatbot

The above fig.3, represents the flow of the Chatbot. The main aim of the proposed work is to find the key state of each conversation made by the customer. That is nothing but identifying the key intent or problem of the customer and reply accordingly so that satisfy the customer's need within a few conversations. In a long conversation between a person and a chatbot, the chatbot needs to remember the context or manage dialogue states. The problem of dialogue management is important to ensure that the communication between people and machine is smooth. The function of dialogue management component is important to receive input from NLU component, to manage dialogue states, dialogue contexts, and to transmit output to Natural Language Generation. Natural Language Generation then generates response to the user's request.

System Implementation

Creating a chatbot is a challenging because chatbot must chat with the user like a human. Communication is easy for human but creating a machine to chat like human is difficult.

To implement the chatbot and compare the state of art, two methods has been implementedand they are

1. Intent Classification

2.Finding Keystate Using Deeppavlov

The first important component of a chatbot is creating the brain i.e. neural network. In neural network the main operation is to get the input, pass it to the network layer and generate the output from fig 4. Hidden layer are set of calculations which can produce different kind of result. Dataset is passed to the layers to train and generate the optimum result. Dataset cannot be directly used for training the model, there are some preprocessing techniques to be undertaken and then further continued for training. 


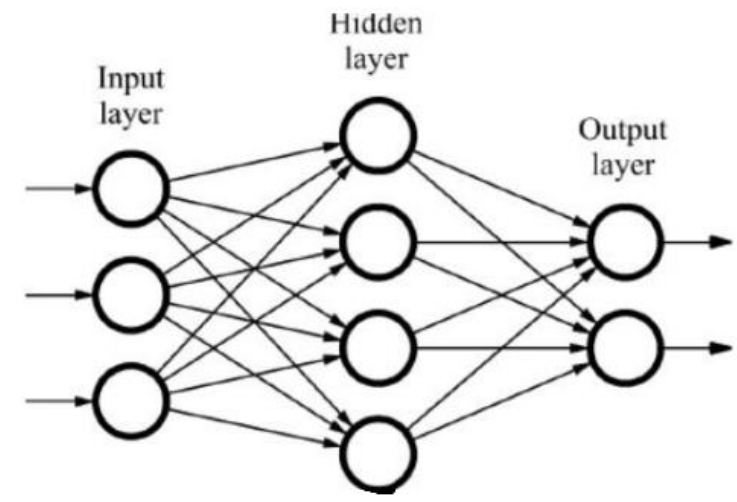

Fig 5.1Neural Network Layer

To develop any model, the first step is to remove the unwanted data and duplicate text in the dataset, only then we can develop an accurate model can be developed to give high accuracy. There are four different parts namely Cleaning, Annotation, Normalization and Analysis.

Cleaning consists of getting rid of the less useful parts of the text through stopword removal, dealing with capitalization and characters and other details. Annotation consists of the application of a scheme to texts. Annotations may include structural markup and part-of-speech (POS) tagging.Analysis consists of statistically probing, manipulating and generalizing from the dataset for feature analysis. Text often has variety of capitalization of reflecting the beginning of sentences, proper nouns emphasis. The most common method or approach is to reduce every sentence in the dataset into lowercase so that the model will predict accurately.

A majority of words in a given text are connecting parts of a sentence rather than showing subjects, objects, or intent. Words like "the" or "and" can be removed by comparing text to a list of stopword.

In developing an intent classification model, the dataset is classified based on intent so that model can be developed with ease and can be trained with high accuracy so that the model can easily identify the customers query based on the intent, so that the customers problem can be solved within few conversations. Chatbot is implemented by two Methods Intent Classification and Finding Keystate using Deeppavlov.

The model which is developed used BERT (Bidirectional Encoder Representations from Transformers) for Natural Language Understanding and benchmark it with LSTM. The reason behind choosing transformer is poor classification result with sequence to sequence models on Intent Classification task when the data is not balanced. So BERT model is chosen because it can even classify intent even on imbalanced dataset. The architecture of intent classification is shown in fig 5 . The step by step process in intent classification model is shown in fig 6 .

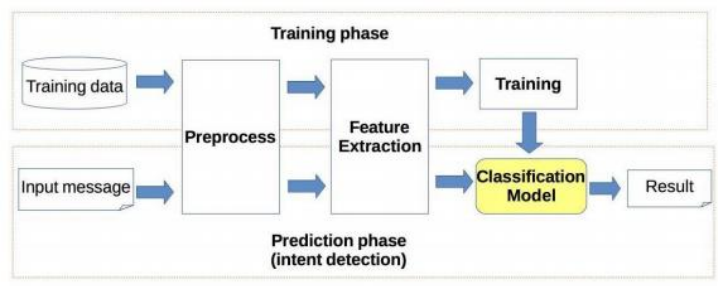

Fig 5 Intent classification

BERT is basically a trained Transformer Encoder stack, with 12 in the base version and 24 in the large version, compared to 6 encoder layers in the original Transformer (LSTM). BERT encoders have larger feedforward networks (768 and 1024 nodes in Base and Large version respectively). The 
main advantage of BERT is that it was trained on Wikipedia and Book Corpus. Its open-sourced model code broke several records for difficult language-based tasks.

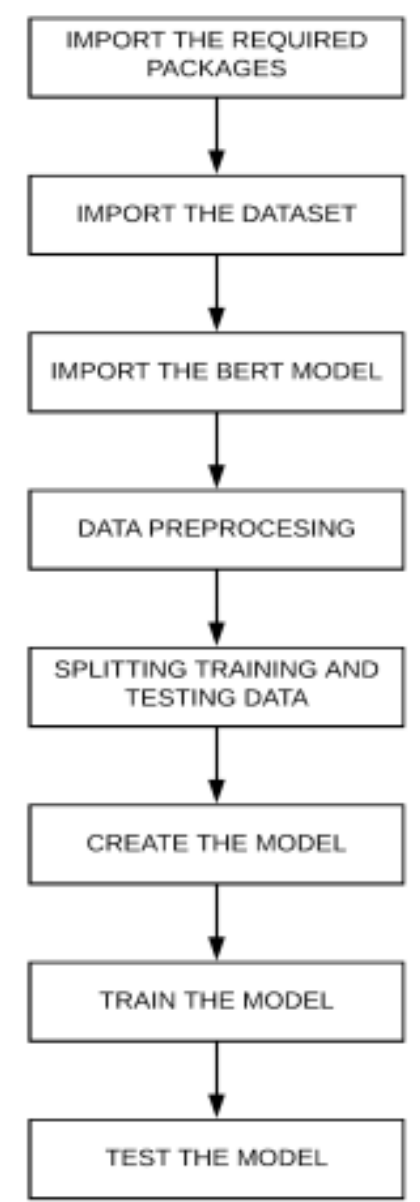

Fig 6 Steps in intent classification model

Finding keystate using deeppavlov

GoBot is a pretrained model in Deeppavlov based on the architecture from the fig 6.0. There are different kinds of GoBot model in Deeppavlov and each of them has its own specification. All the component requires algorithms to implement. NLU is implemented by using Named Entity Recognition (NER) which help us to find to the intent, entities, dialog state.The components or the requirements needed for developing the chatbot isWord Embedding, Named Entity Recognition, Intent classifier and Dataset

To find words that have same meaning which have similar representation by using word embedding. Each word in a document or a sentence will have real-values vectors which willbe defined in vector space. This technique is a breakthrough for deep learning. Because realvalued vectors tend to be in tens or hundreds dimension which is helpful to find similar words. To identify the dialog state, the bot must be able to understand the key state of a sentence, this is done with the help of NER. In 
Deeppavlov there are several models used for Named Entity Recognition (NER). Classifiers will identify the class of an element. Intent Classifier will find the class of each key state and the probability of its occurrence is noted.Dataset reader is initialized to the folder where are all the dataset is recorded.Once dataset_reader is initialized, the dataset iterator is used to iterate throughthe files. With the help of dataset_iterator all the data's in the dataset can be read and userresponse and the system response can be shown separate.After initializing the dataset_reader and dataset_iterator, creation of database is done.

While loading the database it is important to specify the primary key for the database. The primary key will be among the slotfill field name. In this work there is only one slotfill field name which is "problem". If there are many slotfill field names like "area" then primary key must be chosen properly. And now all the db_result which is included in the dataset will be loaded to the database. The next part is to create the slotfill and load the path to the slotfill_configs. Once the file is read from the path, evaluating the model is done and duringevaluating the slot_accuracy is found to be 0.5.Now the slotfill model is built and if any dialog is passed as a parameter to the model,it will return key state of the dialogue.Building the GoBot model is done next. While building the model the path to the database, slotfill, dataset is set to GoBot model. During this stage the type of embedder is fixed to the pipeline path.

The GoBot model is trained while passing the batch_size,max_batches, val_every_n_batches, log_every_n_batches which is shown in the fig 7. While training the model the accuracy is found to be 0.76. This accuracy can be increased while increasing the dataset and batch size. After building the model it is evaluated which is shown in fig 8 and the accuracy is also found to be 0.76 .

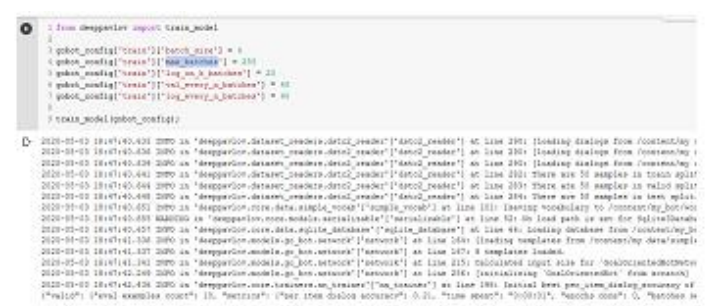

Fig 7 Training the model

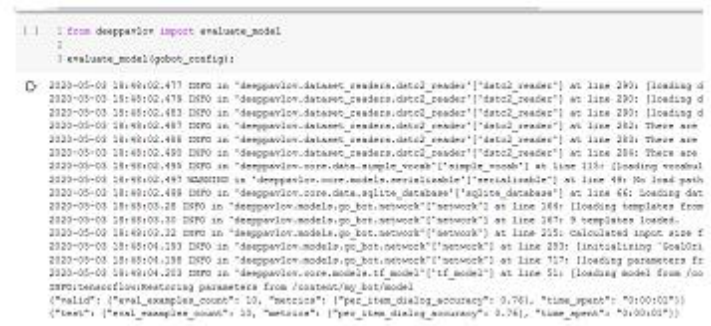

Fig 8 Evaluating the model

Now when the bot is given the queries by the user, the bot will be able to identify the key state with the help of tracker and it shown in the fig 8 and for the query "software for qr code",the tracker is able to find the key state of the dialog which is "QR code" and the corresponding reply is given by the bot. 


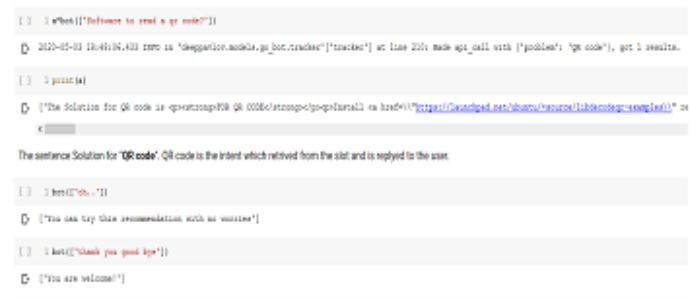

Fig 9 Working of the bot

The results of the two models(Intent Classification and Gobot model) is displayed inthe dashboard and is shown in the fig 10.

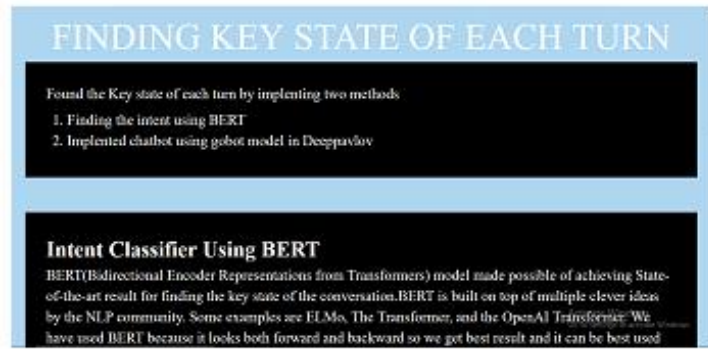

\section{CONCLUSION}

Fig 10 Chatbot Dashboard

The model was created and can be used to identify the intent from the user's problem with the help of intent classification. Intent classification can be helpful to achieve the user's goal within a short conversation. But there will be significant challenge to create a chatbot for a particular task. Intent classification can be integrated to models to build a contextualized chatbot. These types of chatbot can be used in different organization. In customer service companies these type of chatbot will bring much profit and the more important part of these models are the larger the dataset the more the intent classification and chatbot will be accurate.

\section{Reference}

[1] Barker, S., How chatbots help. MHD Supply Chain Solutions, 2017. 47(3): p. 30.

[2] Chen, H., et al., A Survey on Dialogue Systems: Recent Advances and New Frontiers. ACM SIGKDD Explorations Newsletter, 2017. 19(2): p. 25-35

[3] Bahdanau, D., K. Cho, and Y. Bengio, Neural Machine Translation by Jointly Learning to Align and Translate. 2014.

[4] Sutskever, I., O. Vinyals, and Q.V. Le, Sequence to sequence learning with neural networks, in Proceedings of the 27th International Conference on Neural Information Processing Systems Volume 2. 2014, MIT Press: Montreal, Canada. p. 3104-3112.

[5] Shevat, A. (2017). Designing bots: Creating conversational experiences. UK: O'Reilly Media.

[6] Watson, H. J. (2017). Preparing for the cognitive generation of decision support. MIS Quarterly Executive, 16(3), 153-169.

[7] Gnewuch, U., Morana, S., \& Maedche, A. (2017). Towards designing cooperative and social conversational agents for customer service. In: Proceedings of the 38th International Conference on Information Systems (ICIS). Seoul: AISel. 